\title{
ON THE CROSSING NUMBER OF HIGH DEGREE SATELLITES OF HYPERBOLIC KNOTS
}

\author{
ZHENG-Xu He
}

\begin{abstract}
Let $K$ be a hyperbolic knot, and let $K^{\prime}$ be a satellite of $K$ of (homological) degree $p$, where $p$ is an integer. We show that the crossing number of $K^{\prime}$ is at least $(\operatorname{area}(\mathcal{E}))(\operatorname{length}([m]))^{-1}(2 \pi-2 \text { length }([m]))^{-1} p^{2}$, where area $(\mathcal{E})$ is the area of the critical horo-torus of the hyperbolic structure on the knot complement and length $([m])$ is the length of the meridian in the horo-torus. Our estimate is an improvement over an earlier result of M. Freedman and the author in many cases.
\end{abstract}

\section{Introduction}

The crossing number of a knot or link $K$, denoted by $\operatorname{cr}(K)$, is defined to be the minimal number of crossings in a plane diagram representing the knot or link (see e.g., [8, page 8]). For example, the crossing number of the trefoil is 3 , and the figure-eight knot 4. Using Jones polynomial, L. Kauffman [7] has shown that if a knot or link can be represented by a reduced alternating diagram, then its crossing number is actually equal to the number of crossings in the diagram. This has also been extended to semi-alternating links, Montesinos links, and other special cases (see $[9,14]$ ). However, many elementary questions regarding the crossing number remain unanswered. For example, it is believed, but not proved, that the crossing number of a satellite is at least equal to that of its companion (see e.g., [8]).

Let $K$ be a non-trivial knot which is represented by a smooth, simple loop $\kappa$ in $\mathbb{R}^{3}$. Let $\mathcal{T}$ be a compact tubular neighborhood of $\kappa$. That is, $\mathcal{T}$ is the image of a smooth embedding $I: \mathbb{S}^{1} \times \mathbb{D}^{2} \rightarrow \mathbb{R}^{3}$ such that $\kappa=I\left(\mathbb{S}^{1} \times\{0\}\right)$; where $\mathbb{D}^{2}$ denotes the closed unit disk in $\mathbb{R}^{2}$. The degree of an (oriented) loop in $\mathcal{T}$ will mean the homological degree. (This is well-defined up to a sign since $H_{1}(\mathcal{T} ; \mathbb{Z}) \cong \mathbb{Z}$.) Let $p$ be an integer. A knot will be called a degree $p$ satellite of $K$ if it can be represented by a simple loop of degree $\pm p$ in $\mathcal{T}$.

It is conjectured that the crossing number of a degree $p$ satellite $K^{\prime}$ of $K$ is at least $p^{2}$ times the crossing number of $K$. In [4], we have shown that $\operatorname{cr}\left(K^{\prime}\right)$ is bigger than or equal to $p^{2}(2$ genus $(K)-1)$. The proof made an application of works of D. Gabai [5] on foliations of 3-manifolds and of W. Thurston on norms of homology classes [16] (see also [13]).

Received January 15, 1998.

This research is partially supported by an NSF grant. 
Denote $\mathbb{S}^{3}=\mathbb{R}^{3} \cup\{\infty\}, \quad \mathcal{M}(K)=\mathbb{S}^{3}-\mathcal{T}$, and $\overline{\mathcal{M}}(K)=\mathcal{M}(K) \cup \partial \mathcal{T}$. Then the inclusion $\partial \mathcal{T} \subseteq \overline{\mathcal{M}}(K)$ induces an inclusion $\pi_{1}(\partial \mathcal{T}) \subseteq \pi_{1}(\overline{\mathcal{M}}(K))$. Suppose that $K$ is hyperbolic; that is, there exists a complete hyperbolic metric on $\mathcal{M}(K)^{1}$. The group $\pi_{1}(\overline{\mathcal{M}}(K))$ is clearly isomorphic to $\pi_{1}(\mathcal{M}(K))$, and hence acts on the universal cover of $\mathcal{M}(K)$ as a group of hyperbolic isometries. For simplicity of notation, we will just identify the two: $\pi_{1}(\overline{\mathcal{M}}(K))=\pi_{1}(\mathcal{M}(K))$ (the identification is unique up to inner isomorphisms). Let $\mathcal{B}(K)$ be the largest open horoball in the universal cover of $\mathcal{M}(K)$ such that $\mathcal{B}(K) / \pi_{1}(\partial \mathcal{T})$ embedds into $\mathcal{M}(K)$. Let $\partial \mathcal{B}(K)$ be the bounday of $\mathcal{B}(K)$ in the hyperbolic space, and let $\mathcal{E}(K)=\partial \mathcal{B}(K) / \pi_{1}(\partial \mathcal{T})$. The space $\mathcal{E}(K)$, with the induced metric, is a euclidean torus. Moreover, $\pi_{1}(\mathcal{E}(K))$ can be naturally identified with $\pi_{1}(\partial \mathcal{T})$.

The euclidean torus $\mathcal{E}(K)$ is called the critical horo-torus of the hyperbolic manifold $\mathcal{M}(K)$.

Let $m$ and $l$ be a meridian and longitude respectively on $\partial \mathcal{M}(K)=\partial \mathcal{T}$. Thus, $m$ bounds a disk in $\mathcal{T}$, and $l$ bounds a Seifert surface in $\overline{\mathcal{M}}(K)$. For simplicity, we will denote by $[m]$ and $[l]$ the corresponding homology classes in $H_{1}(\mathcal{E}(K) ; \mathbb{Z})=\pi_{1}(\mathcal{E}(K))=\pi_{1}(\partial \mathcal{T}) \cong \mathbb{Z}^{2}$. For any pair of integers $k, j$, let length $(k[m]+j[l])$ denote the length of a geodesic loop in $\mathcal{E}(K)$ representing the homology class $k[m]+j[l]$.

Our main theorem can be stated as follows.

1.1. Theorem. Let $K$ be a hyperbolic knot, and let $p$ be an integer. Let $\mathcal{E}(K)$ be the critical horo-torus of the hyperbolic structure on the knot complement $\mathcal{M}(K)$. The crossing number of any degree $p$ satellite $K^{\prime}$ of $K$ satisfies the following estimate:

$$
\operatorname{cr}\left(K^{\prime}\right) \geq\left(\frac{\operatorname{area}(\mathcal{E}(K))}{\operatorname{length}([m])(2 \pi-\operatorname{length}([m]))}\right) p^{2} .
$$

By a result of M. Gromov and W. Thurston [6], we have length $([m])<2 \pi$ (since otherwise one would be able to construct a metric of nonpositive curvature on $\left.\mathbb{S}^{3}\right)$. On the other hand, it is known that the length of any non-trivial loop in $\mathcal{E}(K)$ is at least 1 (see e.g., [10]). Thus, $1 \leq \operatorname{length}([m])<2 \pi$. It is interesting to note that, by letting $p=1$ in the above theorem, we obtain the following:

$$
\operatorname{length}([m])(2 \pi-\operatorname{length}([m])) \geq \operatorname{area}(\mathcal{E}(K)) / \operatorname{cr}(K) \geq \sqrt{3} / \operatorname{cr}(K),
$$

where the inequality area $(\mathcal{E}(K)) \geq \sqrt{3}$ can be derived by combining a recent result of C. Adams [1] with the classical theorem on the optimal packings of identical circles in the plane. In fact, the following can be proved (see Corollary 3.2$)$ :

$$
\operatorname{length}([m])(2 \pi-\operatorname{length}([m])) \operatorname{cr}(K)-2 \pi \operatorname{length}([m]) \geq \operatorname{area}(\mathcal{E}(K)) \geq \sqrt{3}
$$

\footnotetext{
${ }^{1}$ By Thurston's Geometrization Theorem, a knot is hyperbolic if and only if it is not a nontrivial satellite or a torus knot (see e.g. [15] or [12]).
} 
Theorem 1.1 follows as a corollary of an estimate on the asymptotical crossing number $^{2} \operatorname{ac}(K)$ of $K$ (see Theorem 2.1):

$$
\operatorname{ac}(K) \geq \frac{\operatorname{area}(\mathcal{E}(K))}{\operatorname{length}([m])(2 \pi-\operatorname{length}([m]))} .
$$

Since length $([m])(2 \pi-$ length $([m])) \leq \pi^{2}$, we have

$$
\operatorname{ac}(K) \geq \operatorname{area}(\mathcal{E}(K)) / \pi^{2} .
$$

We do not know under what conditions does area $\left(\mathcal{E}\left(K_{n}\right)\right)$ converge to $\infty$ when $\operatorname{cr}\left(K_{n}\right) \rightarrow \infty$. Where $K_{n}$ is a sequence of hyperbolic knots. One may compare (1.3) with the estimate of $[4]: \operatorname{ac}(K) \geq 2$ genus $(K)-1$. We will see that in some cases, the estimate in the present paper is better.

The paper will be organized as follows. In section 2 , we will recall and discuss the asymptotical crossing number. The proof of our main estimate will occupy section 3. Then, in section 4, we will give an application. Finally in section 5, we will tabulate some computational data for some Montesinos knots, using the program SnapPea, developed by J. Weeks at the Geometry Center, Minnesotta [17].

\section{Asymptotical crossing number}

We will next recall the definition of asymptotical crossing number. The notion was introduced in [4] for estimating the energy of divergence-free vector fields in fluids (or gases) which are subject to incompressible deformations (see also [11, 2, 3] for more background). As in [4], we will give an estimate for the asymptotical crossing number, which implies Theorem 1.1 as an immediate corollary.

Let $L=\left(L_{1}, L_{2}\right)$ be a two-component link. The inter-crossing number of $L$, denoted by $\operatorname{cr}-\mathrm{i}(L)$, is defined to be the minimum number of crossings of the first component over the second in a plane diagram representing the link. Here is a more abstract way to define this. Let $\operatorname{pr}_{12}: \mathbb{R}^{3} \rightarrow \mathbb{R}^{2}$ and $\mathrm{pr}_{3}: \mathbb{R}^{3} \rightarrow \mathbb{R}$ be defined by: $\operatorname{pr}_{12}\left(\left(x_{1}, x_{2}, x_{3}\right)\right)=\left(x_{1}, x_{2}\right)$ and $\operatorname{pr}_{3}\left(\left(x_{1}, x_{2}, x_{3}\right)\right)=x_{3}$. Let $P, Q: \mathbb{S}^{1} \rightarrow \mathbb{R}^{3}$ be a pair of disjoint simple loops. The inter-crossing number of the pair $(P, Q)$ relative to the projection $\operatorname{pr}_{12}$, denoted by $\operatorname{cr}-\mathrm{i}(P, Q)$, is defined to be the cardinality of the set $\left\{(s, t) \in \mathbb{S}^{1} \times \mathbb{S}^{1} ; \operatorname{pr}_{12}(P(s))=\right.$ $\left.\operatorname{pr}_{12}(Q(t)), \operatorname{pr}_{3}(P(s))>\operatorname{pr}_{3}(Q(s))\right\}$. Then inter-crossing number of $\left(L_{1}, L_{2}\right)$ is then the minimum inter-crossing number relative to $\mathrm{pr}_{12}$ of a pair of disjoint simple loops in $\mathbb{R}^{3}$ representing the link.

Let $K$ be a knot, represented by a loop in $\mathbb{R}^{3}$; and let $\mathcal{T}$ be a compact tubular neighbourhood of the loop. Let $p, q$ be integers. A two-component

\footnotetext{
${ }^{2}$ The definition of asymptotical crossing number given in our joint paper [4] is not the same as the one given in Kirby's problem list [8]. Theoretically ours can be smaller, although they are conjectured to be identical.
} 
link $L=\left(L_{1}, L_{2}\right)$ will be called a degree $(p, q)$ satellite link of $K$, if it can be represented by a pair of disjoint simple loops $P, Q: \mathbb{S}^{1} \rightarrow \mathcal{T}$ of degrees $p$ and $q$ respectively. The asymptotical crossing number ac $(K)$ of $K$ is defined to the infimum of $\operatorname{cr}-\mathrm{i}(L) /|p q|$, where $p, q \in \mathbb{Z}-\{0\}$, and $L$ is a degree $(p, q)$ satellite link of $K$.

Clearly, $\operatorname{ac}(K) \leq \operatorname{cr}(K)$. In [4], it is proved that $\operatorname{ac}(K) \geq 2 \operatorname{genus}(K)-1$. The conjecture is that $\operatorname{ac}(K)$ actually equals $\operatorname{cr}(K)$. The method in present paper will prove the following theorem.

2.1. Theorem. If $K$ is a hyperbolic knot as in Theorem 1.1, then

$$
\operatorname{ac}(K) \geq \frac{\operatorname{area}(\mathcal{E}(K))}{\operatorname{length}([m])(2 \pi-\operatorname{length}([m]))} .
$$

In light of the following observation and the fact that $\mathrm{cr} \geq \mathrm{ac}$, it is easy to see that the above theorem implies Theorem 1.1.

2.2. Remark. If $p$ is a non-zero integer, and $K^{\prime}$ a degree $p$ satellite of $K$, then

$$
\operatorname{ac}\left(K^{\prime}\right) \geq p^{2} \operatorname{ac}(K)
$$

\section{Proof of Theorem 2.1}

We will need the following geometrical lemma.

3.1. Lemma. Let $K, \mathcal{T}, \mathcal{M}(K), \overline{\mathcal{M}}(K), \mathcal{E}(K)$ be as in Theorem 1.1. Let $n, r \geq$ 0 and $p \neq 0$ be integers. Let $S$ be a connected compact planar surface with $n+r$ boundary components, and let $F:(S, \partial S) \rightarrow(\overline{\mathcal{M}}(K), \partial \overline{\mathcal{M}}(K))$ be a map such that the homology class $F_{*}[S, \partial S]$ in $H_{2}(\overline{\mathcal{M}}(K), \partial \overline{\mathcal{M}}(K) ; \mathbb{Z}) \cong \mathbb{Z}$ equals $\pm p$ times the generator. Assume that there exist at least $n$ components of $\partial S$ whose image loops under $F$ is homotopically trivial in $\mathcal{T}$. Then

$$
(2 \pi-\operatorname{length}([m])) n+(2 \pi) r \geq 4 \pi+|p| \frac{\operatorname{area}(\mathcal{E}(K))}{\operatorname{length}([m])} .
$$

Proof. For simplicity, we denote $\mathcal{M}=\mathcal{M}(K)$ and $\overline{\mathcal{M}}=\overline{\mathcal{M}}(K)$. By contradiction, let us assume that there exist $n, r, p, S$ and $F$ which satisfy the hypotheses of the lemma, and at the same time (3.1) fails. Let us choose them so that $n+r$ is smallest possible.

If $\gamma:([0,1],\{0,1\}) \rightarrow(S, \partial S)$ is a simple curve joining different boundary components, then the curve $F \gamma:([0,1],\{0,1\}) \rightarrow(\overline{\mathcal{M}}, \partial \overline{\mathcal{M}})$ cannot be homotoped into $(\partial \overline{\mathcal{M}}, \partial \overline{\mathcal{M}})$. Suppose contrarily that it can, then we may cut $S$ along the (non-separating) curve, and obtain a new surface $\tilde{S}$, such that there exists a map $\tilde{F}:(\tilde{S}, \partial \tilde{S}) \rightarrow(\overline{\mathcal{M}}, \partial \overline{\mathcal{M}})$ which equals $F$ outside a small neighborhood of the curve. Let $\tilde{n}, \tilde{r}$ and $\tilde{p}$ be integers such that the hypothesis of the lemma 
is satisfied if $S, F, n, r$ and $p$ are replaced by $\tilde{S}, \tilde{F}, \tilde{n}, \tilde{r}$ and $\tilde{p}$. Clearly, $\tilde{n}+\tilde{r}=n+r-1, \tilde{n} \leq n, \tilde{r} \leq r$, and importantly, $\tilde{p}=p$. Thus, by the minimality of $n+r$, we deduce that (3.1) holds for $\tilde{n}, \tilde{r}, \tilde{p}$ :

$$
(2 \pi-\operatorname{length}([m])) \tilde{n}+(2 \pi) \tilde{r} \geq 4 \pi+|\tilde{p}| \frac{\operatorname{area}(\mathcal{E}(K))}{\operatorname{length}([m])}
$$

But this implies (3.1), a contradiction.

$F$ maps every essential simple loop in $S$ to an essential curve in $\overline{\mathcal{M}}$. If not, we may cut $S$ along the simple loop, and then glue a disk (along the cut) on each component to obtain two compact planar surfaces $S_{1}$ and $S_{2}$. For each $i=1,2$, there is a map $F_{i}:\left(S_{i}, \partial S_{i}\right) \rightarrow(\overline{\mathcal{M}}, \partial \overline{\mathcal{M}})$ which agrees with $F$ outside of the glued disk. There exist integers $n_{i}, r_{i}, p_{i}$ such that $S_{i}, F_{i}$ satisfy the hypothesis of the lemma when $n, r, p$ are replaced by $n_{i}, r_{i}, p_{i}$ respectively; and moreover, $n_{1}+r_{1}+n_{2}+r_{2}=n+r, n_{1}+n_{2}=n$, and $p_{1}+p_{2}=p$. As $n_{i}+r_{i}<n+r$, by minimality of $n+r,(3.1)$ holds for $n_{i}, r_{i}, p_{i}$; i.e.,

$$
(2 \pi-\operatorname{length}([m])) n_{i}+(2 \pi) r_{i} \geq 4 \pi+\left|p_{i}\right| \frac{\operatorname{area}(\mathcal{E}(K))}{\operatorname{length}([m])}
$$

Adding the above inequalities over $i=1,2$, we obtain:

$$
(2 \pi-\operatorname{length}([m]))\left(n_{1}+n_{2}\right)+(2 \pi)\left(r_{1}+r_{2}\right) \geq 8 \pi+\left(\left|p_{1}\right|+\left|p_{2}\right|\right) \frac{\operatorname{area}(\mathcal{E}(K))}{\operatorname{length}([m])} .
$$

It follows that (3.1) holds for $n, r, p$, a contradiction.

Since $K$ is a nontrivial knot and $p \neq 0, n+r$ is at least 3 . The interior $\stackrel{\circ}{S}$ of $S$ admits hyperbolic structures. By small perturbation, we may assume that $F$ is transversal to $\partial \overline{\mathcal{M}}$. Thus $F$ restricts to a map from $\stackrel{\circ}{S}$ into the hyperbolic manifold $\mathcal{M}$. Let $T$ be an ideal triangulation of $\stackrel{\circ}{S}$ (in some hyperbolic metric) such that each ideal edge joins different ends. Then using properties of $F$, we may deform the map such that its restriction to $\stackrel{\circ}{S}$ maps any ideal edge in $T$ to a geodesic curve in $\mathcal{M}$, and any ideal triangle to a (singular) ideal triangle in $\mathcal{M}$. We will still use $F$ to denote the new map.

There is a (unique) metric of curvature $=-1$ on $\stackrel{\circ}{S}$ such that $F$ is length preserving from $\stackrel{\circ}{S}$ to $F(\stackrel{\circ}{S})$. Moreover, it is elementary to show that the metric is complete (because $\mathcal{M}$ is complete and $F(\stackrel{\circ}{S})$ is a closed subset). By Euler characteristic considerations, the area of $\stackrel{\circ}{S}$ is this metric is $2 \pi(n+r-2)$. In the following, we willl let $\stackrel{\circ}{S}$ be endowed with this metric.

Consider the embedded image $\mathcal{B} / \pi_{1}(\partial \mathcal{T}) \subseteq \mathcal{M}$; where we recall that $\mathcal{B}=$ $\mathcal{B}(K)$ is the maximum open horoball in the universal cover of $\mathcal{M}$ such that 
$\mathcal{B} / \pi_{1}(\partial \mathcal{T})$ embedds into $\mathcal{M}$. For each boundary component $C$ of $S$, let $A_{C} \subset \stackrel{\circ}{S}$ be the connected component of $F^{-1}\left(\mathcal{B} / \pi_{1}(\partial \mathcal{T})\right)$ whose closure in $S$ contains $C$.

If $C_{1}$ and $C_{2}$ are different components of $\partial S$, then $A_{C_{1}}$ and $A_{C_{2}}$ are different (hence disjoint). In fact, if $A_{C_{1}}=A_{C_{2}}$, then there is a simple path $\gamma$ in $S$ joining $C_{1}$ to $C_{2}$, such that the interior of $\gamma$ is contained in $A_{C_{1}}=A_{C_{2}} \subset \stackrel{\circ}{S}$, and that $F \gamma$ is a curve whose interior is contained in $\mathcal{B}(K) / \pi_{1}(\partial \mathcal{T}) \subset \mathcal{M}$. This means that $F \gamma$ can be homotoped into the boundary $\partial \overline{\mathcal{M}}$, a contradiction.

The area of $F\left(A_{C}\right)$ is at least equal to length $\left(\left[\left.F\right|_{C}\right]\right)$, the length of a geodesic loop in $\mathcal{E}(K)$ which is homotopic to $\left.F\right|_{C}$ in $\overline{\mathcal{M}}$. This can be proved as follows. First, we may use the upper half space model $\mathbb{H}^{3}=\left\{\left(x_{1}, x_{2}, x_{3}\right) ; x_{3}>0\right\}$ for the universal cover of $\mathcal{M}$, so that $\mathcal{B}(K)=\left\{x_{3}>1\right\}$, and that the action by the homotopy class (in $\pi_{1}(\partial \mathcal{T}) \subset \pi_{1}(\mathcal{M})$ ) of $F_{C}$ is

$$
H_{C}\left(\left(x_{1}, x_{2}, x_{3}\right)\right)=\left(x_{1}+\text { length }\left(\left[\left.F\right|_{C}\right]\right), x_{2}, x_{3}\right) .
$$

For any $t \geq 1$, consider the length $L(t)$ of the set $\left\{\left(x_{1}, x_{2}, x_{3}\right) \in F\left(A_{C}\right) ; x_{3}=t\right\}$. Clearly, the set contains a curve (or a union of curves) which is homologic to $\left.F\right|_{C}$, we deduce that $L(t) \geq \operatorname{length}\left(\left[\left.F\right|_{C}\right]\right) / t$. It follows that the area of $F\left(A_{C}\right)$ is at least $\int_{1}^{\infty}\left(\right.$ length $\left.\left(\left[\left.F\right|_{C}\right]\right) / t\right) d t / t=$ length $\left(\left[\left.F\right|_{C}\right]\right)$. We thus obtain

$$
2 \pi(n+r-2)=\operatorname{area}(F(\stackrel{\circ}{S})) \geq \sum_{C} \operatorname{area}(F(C)) \geq \sum_{C} \operatorname{length}\left(\left[\left.F\right|_{C}\right]\right) .
$$

For each boundary component $C$, let $\alpha_{C}, \beta_{C}$ be such that $\left.F\right|_{C}$ is a curve in $\partial \mathcal{T}$ homologic to $\alpha_{C}[m]+\beta_{C}[l]$. The curve $\left.F\right|_{C}$ is contractible in $\mathcal{T}$ if and only if $\beta_{C}=0$; and in this case,

$$
\operatorname{length}\left(\left[\left.F\right|_{C}\right]\right)=\left|\alpha_{C}\right| \text { length }([m]) \geq \operatorname{length}([m]) .
$$

On the other hand, if $\beta_{C} \neq 0$, then

$$
\operatorname{length}\left(\left[\left.F\right|_{C}\right]\right) \geq\left|\beta_{C}\right| \inf _{j \neq 0} \frac{\operatorname{length}(j[l]+k[m])}{|j|}=\left|\beta_{C}\right| \frac{\operatorname{area}(\mathcal{E}(K))}{\operatorname{length}([m])} .
$$

By the assumption of the lemma, there are at least $n$ components (of $\partial S$ ) $C$ 's for which $\beta_{C}=0$. Moreover, $\sum_{C} \beta_{C}=p$. Thus, using (3.3), (3.4) and (3.5), we deduce that

$$
\begin{aligned}
2 \pi(n+r-2) & \geq \sum_{C} \text { length }\left(\left[\left.F\right|_{C}\right]\right) \\
& \geq \sum_{\beta_{C}=0} \operatorname{length}([m])+\sum_{\beta_{C} \neq 0}\left|\beta_{C}\right| \operatorname{area}(\mathcal{E}(K)) / \operatorname{length}([m]) \\
& \geq n \text { length }([m])+|p| \operatorname{area}(\mathcal{E}(K)) / \operatorname{length}([m]) .
\end{aligned}
$$


This proves the lemma.

Proof of Theorem 2.1. We will first follow the outline of [4]. The difference is that here we will analyze surface areas instead of Thurston norms of homology classes. Let $P: \mathbb{S}^{1} \rightarrow \mathcal{T} \subset \mathbb{R}^{3}$ be a loop of degree $p$. Define $\tilde{P}: \mathbb{S}^{1} \times[0, \infty) \rightarrow \mathbb{R}^{3}$ by $\tilde{P}(s, t)=P(s)-(0,0, t)$. The image of $\tilde{P}$ is a singular cylinder with boundary curve $P\left(\mathbb{S}^{1}\right)$, and (by slightly perturbing $\mathcal{T}$ if necessary) we may assume that $\tilde{P}$ is transverse to $\partial \mathcal{T}$. Let $n$ be the number of components $D_{1}, \ldots, D_{n}$ of $\tilde{P}^{-1}(\mathcal{T})$ which represent nonzero elements (under $\left.\tilde{P}_{*}\right)$ in $H_{2}(\mathcal{T}, \partial \mathcal{T} ; \mathbb{Z}) \cong \mathbb{Z}$. (Note that the component of $\tilde{P}^{-1}(\mathcal{T})$ containing $\mathbb{S}^{1} \times\{0\}$ is not a relative cycle, and is therefore not among the $D_{i}$ 's).

Let $Q: \mathbb{S}^{1} \rightarrow \mathcal{T}$ be a loop of degree $q$. Then for each $i, D_{i}$ will meet the image of $Q$ at least $|q|$ times. It is intuitive to see that the inter-crossing number relative to $\operatorname{pr}_{12}$ of $P$ and $Q$ is at least $|q| n$. As the choices of $\kappa$ and $\mathcal{T}$ are arbitrary, Theorem 2.1 is clearly implied by the following inequality:

$$
n \geq|p| \frac{\operatorname{area}(\mathcal{E}(K))}{\operatorname{length}([m])(2 \pi-\text { length }([m]))} .
$$

In order to prove the above inequality, let $X: \mathbb{D}^{2}-\{0\} \rightarrow \mathbb{S}^{1} \times[0, \infty)$ be defined by

$$
X\left(\left(x_{1}, x_{2}\right)\right)=\left(\left(\frac{x_{1}}{\sqrt{x_{1}^{2}+x_{2}^{2}}}, \frac{x_{2}}{\sqrt{x_{1}^{2}+x_{2}^{2}}}\right) ;-\log \left(x_{1}^{2}+x_{2}^{2}\right)\right),
$$

and let $F_{1}: \mathbb{D}^{2} \rightarrow \mathbb{S}^{3}=\mathbb{R}^{3} \cup\{\infty\}$ be the following map:

$$
F_{1}(x)=\left\{\begin{array}{l}
\tilde{P}(X(x)), \text { for } x \neq 0 \\
\infty, \text { for } x=0
\end{array}\right.
$$

Let $S=\tilde{P}^{-1}(\overline{\mathcal{M}}(K))$, and $F=\left.F_{1}\right|_{S}: S \rightarrow \overline{\mathcal{M}}(K)$. Clearly, $S$ is a compact (oriented) planar surface and $F(\partial S) \subset \partial \overline{\mathcal{M}}(K)$. Moreover, the homology class $F_{*}[S, \partial S]$ in $H_{2}(\overline{\mathcal{M}}(K), \partial \overline{\mathcal{M}}(K) ; \mathbb{Z}) \cong \mathbb{Z}$ equals $\pm p$ times the generator.

By an elementary surgery technique, it is possible to change $S, F$, such that each boundary component of $S$ is mapped by $F$ to a nontrivial curve in $\partial \overline{\mathcal{M}}$, and that no component of $S$ is an annulus. We will also drop those components $T$ of $S$, with the property that $F$ maps each component of $\partial T$ into a loop which is homotopically trivial in $\mathcal{T}$.

Let $S_{u}, u=1,2, \ldots, U$, be the connected components of $S$; where $U \geq 1$. For each $u$, let $n_{u}$ be the number of components of $\partial S_{u}$ of the form $\partial D_{i} ; r_{u}+n_{u}$ the total number of components of $\partial S_{u}$; and $p_{u}$ the integer such that the homology class of $F_{*}\left[S_{u}, \partial S_{u}\right]$ in $H_{2}(\mathcal{M}(K), \partial \mathcal{M}(K) ; \mathbb{Z})$ equals $p_{u}$ times the generator. We have $n=\sum_{u} n_{u}$ and $\pm p=\sum_{u} p_{u}$. By Lemma 3.1, we deduce that whenever $p_{u} \neq 0$,

$$
(2 \pi-\operatorname{length}([m])) n_{u}+(2 \pi) r_{u} \geq 4 \pi+\left|p_{u}\right| \frac{\operatorname{area}(\mathcal{E}(K))}{\operatorname{length}([m])}
$$


But if $p_{u}=0$, then there must be zero, or at least two components of $\partial S_{u}$ which are mapped by $F$ to loops which are not homotopically trivial in $\mathcal{T}$. Zero is ruled out by our assumption, thus $r_{u} \geq 2$, and (3.7) still holds. Sum (3.7) over the $u$ 's, we obtain:

$$
(2 \pi-\operatorname{length}([m])) \sum_{u} n_{u}+(2 \pi) \sum_{u} r_{u} \geq 4 \pi U+\sum_{u}\left|p_{u}\right| \frac{\operatorname{area}(\mathcal{E}(K))}{\operatorname{length}([m])} .
$$

Thus,

$$
(2 \pi-\operatorname{length}([m])) n+(2 \pi) \sum_{u} r_{u} \geq 4 \pi U+|p| \frac{\operatorname{area}(\mathcal{E}(K))}{\operatorname{length}([m])}
$$

By an elementary topological argument, it is easy to show that $\sum_{u=1}^{U} r_{u} \leq$ $2 U-1$. So (3.8) implies:

$$
(2 \pi-\operatorname{length}([m])) n+2 \pi(2 U-1) \geq 4 \pi U+|p| \frac{\operatorname{area}(\mathcal{E}(K))}{\operatorname{length}([m])} .
$$

As a consequence,

$$
(2 \pi-\operatorname{length}([m])) n \geq 2 \pi+|p| \frac{\operatorname{area}(\mathcal{E}(K))}{\operatorname{length}([m])} .
$$

Thus (3.6), and hence Theorem 2.1 follows.

3.2. Corollary. Let $K, \mathcal{T}, \mathcal{M}(K), \overline{\mathcal{M}}(K), \mathcal{E}(K)$ be as in Theorem 2.1. We have,

$$
\begin{aligned}
\operatorname{length}([m])(2 \pi-\operatorname{length}([m])) \operatorname{cr}(K)-2 \pi \operatorname{length}([m]) \geq & \\
& \operatorname{area}(\mathcal{E}(K)) \geq \sqrt{3} .
\end{aligned}
$$

Proof. In the proof of Theorem 2.1, let $p=q=1$, and let $\kappa$ be a loop representing $K$ such that the projected image $\operatorname{pr}_{12} \kappa$ in $\mathbb{R}^{2}$ has exactly $\operatorname{cr}(K)$ crossings (i.e., double points). Let $P=\kappa$, and $Q$ a parallel copy of $P$ (nearby in space), so that $\operatorname{cr}-\mathrm{i}(P, Q)=\operatorname{cr}(K)$. Let $n$ be as in the proof, we then have $n \leq \operatorname{cr}-\mathrm{i}(P, Q)=$ $\operatorname{cr}(K)$. Thus, as $p=1,(3.10)$ follows directly by (3.9).

\section{An application}

We will relate our main estimate to some results and conjectures in $[4]^{3}$.

\footnotetext{
${ }^{3} \mathrm{By}$ the way, in the paragraph just before Appendix $\mathrm{A}$ in the paper, on page 224, the sentence "Recalling that power $=($ voltage $)(\text { resistance })^{-2}$, we see that it will require at least $(16 / \pi)^{1 / 3}$ mega watts of power to maintain a one volt per revolution drop in electrical potential" needs to be replaced by "Recalling that power $=($ resistance $)$ (current) $)^{2}$, we see that it will require at least $16 / \pi)^{1 / 3}$ mega watts of power to maintain a revolutionary electrical flow of flux equal to 1 Ampère.
} 
Let $K$ be a knot. Consider a solid torus $\mathcal{T}$ of knot type $K$ made by 1 gram of copper. How much power is it needed to maintain a steady revolutionary ellectric flow of flux (or current) equal to one Ampère? The power needed (measured in watts) will be denoted by $\mathrm{R}(\mathcal{T})$; and we will say that the electrical resistance of $\mathcal{T}$ is $\mathrm{R}(\mathcal{T})$ ohms. Here it is helpful to recall the high school Physics formula: power is equal to resistance times current squared.

Assume that the solid torus has flexible shape, but fixed knot type ${ }^{4}$. If the knot type is trivial, it is possible to deform $\mathcal{T}$ (while keeping its volume and knot type fixed) in such a way that $\mathrm{R}(\mathcal{T})$ becomes arbitrarily small (see [4]). This phenomenon is prevented if $K$ is a nontrivial knot. In fact, by $[4,(0.1),(2.40)$, (3.4)], we have,

$$
\mathrm{R}(\mathcal{T}) \geq \mu(16 / \pi)^{1 / 3} \operatorname{ac}(K) ;
$$

where $\mu$ is the electrical resitance between a pair of opposite faces in a (solid) cube made by 1 gram of copper.

It was conjectured [4] that the resistance of $\mathcal{T}$ becomes arbitrarily large if its knot type gets more and more complicated; that is, if $\mathcal{T}_{n}$ is a sequence of solid tori (each one is made by 1 gram of copper) such that the crossing numbers of their knot types diverges to $\infty$, then $\mathrm{R}\left(\mathcal{T}_{n}\right) \rightarrow \infty$. In case when the knot types $K_{n}$ of $\mathcal{T}_{n}$ are all hyperbolic, we have by (4.1) and (1.4) that

$$
\mathrm{R}(\mathcal{T}) \geq \mu(16 / \pi)^{1 / 3} \operatorname{area}\left(\mathcal{E}\left(K_{n}\right)\right) / \pi^{2} .
$$

We hope that the above inequality, combined with other results, can be helpful in solving the conjecture.

Finally, we note that similar results hold for hyperbolic links.

\section{Numerical data}

Using SnapPea, we have obtained some concrete data for some randomly chosen Montesinos knots, and for which the estimate of our main theorem is better than the earlier estimate $\operatorname{ac}(K) \geq 2 \operatorname{genus}(K)-1$.

Let $a, b, c$ be positive odd integers. The Montesinos knot $M(a, b, c)$ is the knot represented in Figure 5.1, where $a, b, c$ are the numbers of half-turns.

It is easy to see that the genus of $M(a, b, c)$ is equal to 1 , so the estimate of [4] says that $\operatorname{ac}(M(a, b, c)) \geq 1$. We have computed the euclidean structure of the critical torus $\mathcal{E}(M(a, b, c))$ for some values of $a, b, c$, and we see from these examples that the new estimate is an improvement.

Example 1. $(a, b, c)=(7,3,5)$ : The actions of $[m]$ and $[l]$ are given by the following translations of $\mathbb{R}^{2}$ :

$$
\begin{aligned}
& {[m](x, y)=(x-0.233762, y+1.763538)} \\
& {[l](x, y)=(x+5.767326, y) .}
\end{aligned}
$$

\footnotetext{
${ }^{4}$ This is the same as saying that $\mathcal{T}$ can be deformed by a volume-preserving diffeomorphism of $\mathbb{R}^{3}$.
} 


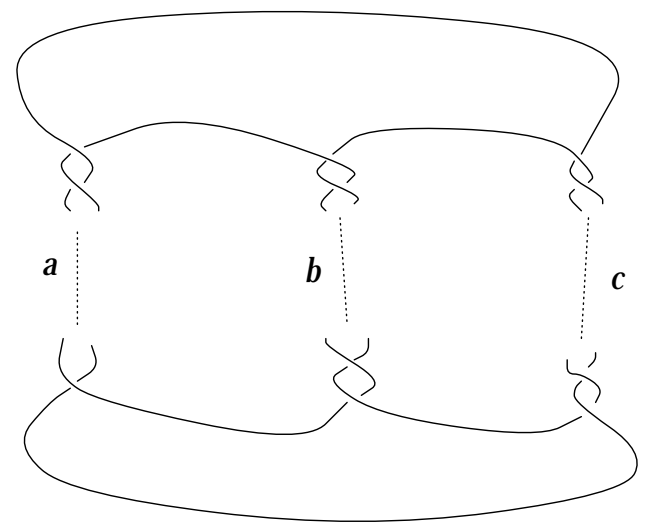

Figure 5.1

We obtain $\operatorname{area}(\mathcal{E})=10.1709, \operatorname{length}([m])=1.77896$, and thus,

$$
\operatorname{ac}(M(7,3,5)) \geq 1.26933 \text {. }
$$

Example 2. $(a, b, c)=(9,7,7)$ :

$$
\begin{aligned}
& {[m](x, y)=(x-0.079160, y+1.863726)} \\
& {[l](x, y)=(x+5.955076, y) .}
\end{aligned}
$$

We obtain $\operatorname{area}(\mathcal{E})=11.0986$, length $([m])=1.86541$, and thus,

$$
\operatorname{ac}(M(9,7,7)) \geq 1.34676 .
$$

Example 3. $(a, b, c)=(19,11,13)$ :

$$
\begin{aligned}
& {[m](x, y)=(x-0.017660, y+1.927930)} \\
& {[l](x, y)=(x+5.934772, y) .}
\end{aligned}
$$

We obtain area $(\mathcal{E})=11.4418$, length $([m])=1.92801$, and thus,

$$
\operatorname{ac}(M(19,11,13)) \geq 1.36263 .
$$

Example 4. $(a, b, c)=(29,23,25)$ :

$$
\begin{aligned}
& {[m](x, y)=(x-0.002466, y+1.981812)} \\
& {[l](x, y)=(x+5.990580, y) .}
\end{aligned}
$$

We obtain $\operatorname{area}(\mathcal{E})=11.8722$, length $([m])=1.98243$, and thus,

$$
\operatorname{ac}(M(29,23,25)) \geq 1.39248 \text {. }
$$

\section{Acknowledgement}

The author is indebted to Professor Michael Freedman for initiating the project, and sharing many of his ideas, including those contained in our joint paper [4]. 


\section{References}

1. C. Adams, The noncompact hyperbolic 3-manifold of minimal volume, Proc. Amer. Math. Soc. 100 (1987), 601-606.

2. V. I. Arnold, The asymptotical Hopf invariant and its applications, Sel. Math. Sov 5 (1986), 327-345.

3. V. I. Arnold and B. A. Khesin, Topological methods in hydrodynamics, Annual review of fluid mechanics, Vol. 24, Annual Reviews, Palo Alto, CA, 1992, pp. 145-166.

4. M. H. Freedman and Z.-X. He, Divergence-free fields: energy and asymptotical crossing number, Ann. of Math. 134 (1991), 189-229.

5. D. Gabai, Foliations and the topology of 3-manifolds, J. Diff. Geom. 18 (1983), 445-503.

6. M. Gromov and W. P. Thurston, Pinching constants for hyperbolic manifolds, Invent. Math. 89 (1987), 1-12.

7. L. H. Kauffman, State models and the Jones polynomial, Topology 26 (1987), 395-407.

8. R. Kirby, Problems in low-dimensional topology, Dec. 1995.

9. W. Lickorish and M. B. Thistlethwaite, Some links with non-trivial polynomials and their crossing-numbers, Comment. Math. Helv. 63 (1988), 527-539.

10. R. Meyerhoff, Sphere packing and volume in hyperbolic 3-space, Comment. Math. Helv. 61 (1986), 271-278.

11. H. K. Moffatt, Magnetostatic equilibra and analogous Euler flows of arbitrary complex topology, Part 1. fundamentals, J. Fluid Mech. 159 (1985), 359-378.

12. J. Morgan, On Thurston's uniformization theorem for three-dimensional manifolds, The Smith Conjecture, edited by H. Bass and J. Morgan, 1984, pp. 37-125.

13. M. Scharlemann, Sutured manifolds and generalized Thurston norms, J. Diff. Geom. 29 (1989), 557-614.

14. M. B. Thistlethwaite, On the Kauffmann polynomial of an adequate link, Invent. Math. 93 (1988), 285-296.

15. W. P. Thurston, Hyperbolic structures on 3-manifolds, I, II, III, Part I was published in Ann. of Math., 124 (1986), 203-246.

16. __ Norm for the homology of 3-manifolds, Mem. Amer. Math. Soc. 339 (1988), 99-130.

17. J. Meeks, SnapPea, a computer program for creating and studying hyperbolic 3-manifolds, available from www.geom.umn.edu.

Dept. of Mathematics, U.C. San Diego, La Jolla, CA 92093-0112

E-mail address: zhe@math.ucsd.edu 